\title{
Die Beatmung des ARDS-Patienten - Kunst oder Wissenschaft?
}

\author{
Tobias Welte
}

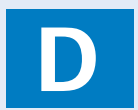

ie Veröffentlichung der ersten ARDS Network Study im Jahre 2000 eröffnete ein neues Zeitalter klinischer Studien für diese schwere Erkrankung. Damit verbunden war die Hoffnung, die optimale Beatmungseinstellung herauszufinden und so eine Reduktion von Morbidität, Letalität und der bei ARDS enormen Kosten der Behandlung zu erreichen. 6 Jahre später ist eine gewisse Ernüchterung eingetreten, weil sich viele Dinge als komplizierter als erwartet gezeigt haben und die Fortschritte gerade im Hinblick auf ein verbessertes Überleben der Patienten nur gering sind.

Die ARDS Network Study konnte überzeugend belegen, dass eine als „protektive Beatmung“ bezeichnete Strategie mit einem niedrigen Atemzugvolumen von $6 \mathrm{ml} /$ kg Körpergewicht im Vergleich zu einer klassischen Strategie mit $12 \mathrm{mg} / \mathrm{kg}$ Körpergewicht zu einem verbesserten Überleben von Patienten beiträgt. Dies wurde inzwischen in anderen Studien bestätigt. Pathophysiologisch lassen sich dafür viele Gründe finden. So führt die Überdehnung von Alveolen zu einer strukturellen Schädigung verschiedener für die Funktion der Lunge wichtigen Zellpopulationen wie beispielsweise der Surfactant produzierenden Alveolarzellen Typ II oder der Epithelzellen. Die Dehnung von Geweben induziert eine sowohl pro- als auch antiinflammatorisch überschießende Antwort, die sowohl zur Schädigung pulmonaler Strukturen beiträgt als auch eine wesentliche Rolle für die Entwicklung des Multiorganversagens trägt. Reduktion der Scherkräfte und Überdehnungsvorgänge trägt sowohl mechanisch wie biochemisch zu einer Verringerung der pulmonalen Schädigung bei und verbessert die Prognose der Patienten.

Die protektive Beatmung wurde daher in praktisch allen intensivmedizinischen Leitlinien verankert und auf unzähligen Kongressen vorgestellt und verfestigt. Nichtsdestotrotz zeigte die Prävalenzstudie des Kompetenznetzwerks Sepsis, dass weniger als $10 \%$ der Patienten leitliniengerecht beatmet werden, obwohl den
Behandlern die Richtlinien bestens bekannt waren. Ähnlich schlechte Ergebnisse aus USA wurden damit begründet, dass die Diagnose des akuten Lungenversagens und des ARDS häufig zu spät gestellt und die protektive Strategie alleine für die am schwersten kranken Patienten reserviert wird.

Daneben dürfte jedoch eine Rolle spielen, dass viele Fragen der Beatmungseinstellung weitgehend ungeklärt sind. So konnten die genannten Studien zwar zeigen, dass $6 \mathrm{ml} / \mathrm{kg}$ Körpergewicht zu besseren Ergebnissen führt als hohe Atemzugvolumina. Ungeklärt blieb jedoch, ob eine weitere Erniedrigung des Zugvolumens und des Plateau-Drucks nicht zu einer weiteren Verbesserung der Prognose der Patienten beitragen kann. Eine neuere Studie zeigt, dass in komplett dys- bis atelektatischen Lungenbezirken bereits kleine Atemzugvolumen ausreichen, um eine nennenswerte Hyperventilation zu erreichen. Andererseits tragen zu kleine Atemzugvolumen zu einer erheblichen Hyperkapnie bei, die aufgrund ihrer Einflüsse auf die Hämodynamik - vor allem im cerebrovaskulären Bereich als durchaus problematisch angesehen wird. Möglicherweise ermöglicht der Einsatz des extrakorporalen Lung Assist (ECLA) mit seiner hohen $\mathrm{CO}_{2}$-Eliminationspotenz in Zukunft niedrigere Atemzugvolumen bei vertretbaren $\mathrm{pO}_{2}$-Werten, erste Fallberichte für verschiedene Indikationen deuten daraufhin. In ähnlicher Weise reduziert auch die Hochfrequenzventilation die Atemzugvolumina und damit den potentiellen Lungenschaden. Kontrollierte Studien fehlen jedoch für beide Verfahren.

Während in der Frage des richtigen Atemzugvolumens zumindest tendenziell Einigung herrscht, ist die Frage des richtigen endexspiratorischen Drucks (PEEP) zur Vermeidung von erneutem Alveolarkollaps in der Exspirationsphase gänzlich ungeklärt. Etabliert hatte sich in den letzten Jahren das in der ARDS Network Study verwendete Protokoll zur Einstellung des PEEP in Abhängigkeit von Atemzugvolumen und Oxigenierung des Patienten. Überraschend war allerdings, dass 
das mittlere PEEP-Niveau in allen Studien, die mit diesem Protokoll arbeiteten, deutlich unter $10 \mathrm{~cm} \mathrm{H}_{2} \mathrm{O}$ lag, auch wenn es sich um sehr schwere kranke Patienten mit hoher Letalität handelte. Eine weitere Networkstudie zur Ermittlung des optimalen PEEP-Niveaus konnte allerdings durch Einsatz höherer PEEP-Werte keine Verbesserung der Prognose von ARDS-Patienten erreichen. Auch der Versuch, mit hohen in- und exspiratorischen Drücken über kurze Zeiträume eine Eröffnung der Alveolen und damit eine Rekrutierung von Gasaustauschfläche zu erreichen, erbrachte keine wirklichen Vorteile. Eine gerade von L. Gattinoni im New Engl J Med veröffentlichte Studie bietet eine interessante Erklärung für die kontroversen Ergebnisse verschiedener Studien im Hinblick auf PEEP an. Er konnte zeigen, dass sich die Möglichkeit der Alveolenrekrutierung von ARDS- Patient zu ARDS-Patient, ja sogar zwischen verschiedenen Arealen einer Lunge dramatisch unterscheidet. Überraschenderweise ist jedoch das Überleben nicht besser, wenn viele gut rekrutierbare Areale vorhanden sind, im Gegenteil sind langfristig gesehen die Patienten im Vorteil, bei denen eine Rekrutierung nicht sofort gelingt. Wahrscheinlich sind die schlecht rekrutierten Areale vor Überblähung und damit struktureller Schädigung geschützt und erholen sich bei einer Besserung der Gesamtsituation des Patienten vollständiger. Die alte Prämisse, dass eine Rekrutierung um jeden Preis, eine schnelle Verbesserung der Oxigenierungssituation des Patienten also das Primärziel der Beatmungstherapie seien, muss damit erheblich in Frage gestellt werden.

Für die Zukunft ergeben sich damit vollkommen neue Konzepte der Beatmungstherapie von ARDS-Patienten. Natürlich muss ein Gasaustausch in der Weise garan- tiert werden, dass es mit dem Leben vereinbar ist. Oberstes Ziel der Beatmungstherapie muss dabei jedoch die Reduktion des beatmungsbedingten Lungenschadens und seiner Konsequenzen für den Gesamtorganismus. Ein generelles Konzept zur Erreichung dieses Ziels steht im Moment nicht zur Verfügung. In Studien eingesetzte diagnostische Möglichkeiten (CT unter verschiedenen Beatmungsmanövern bei Gattinoni) ist in der Praxis nicht durchführbar. Die Umsetzung des wenigen Etablierten (niedriges Atemzugvolumen) und die genaue Beobachtung und daran orientiert individuelle Einstellung der Beatmungsparameter rückt wieder in den Vordergrund. Dafür braucht es erfahrene Behandler und gute klinische und pathophysiologische Grundkenntnisse. Beatmung wird damit vielleicht wieder mehr Kunst und nicht bis ins letzte abgesicherte wissenschaftliche Evidenz. Beatmung braucht damit auch den erfahrenen Arzt und ist für Checklistenmedizin denkbar ungeeignet.

Das ist vielleicht nicht die schlechteste Botschaft der derzeitigen Debatte.

\section{Korrespondenzadresse}

Prof. Dr. Tobias Welte

Abteilung Pneumologie

Medizinische Hochschule Hannover

Carl-Neuberg-Str. 1

30659 Hannover

Telefon: 0511/532-3531

Telefax: 0511/532-3353

E-mail: welte.tobias@mh-hannover.de 